\title{
Electroweak vacuum collapse induced by vacuum fluctuations of the Higgs field around evaporating black holes
}

\author{
Kazunori Kohri ${ }^{1,2,3, *}$ and Hiroki Matsui ${ }^{1,2, \dagger}$ \\ ${ }^{1}$ KEK Theory Center, IPNS, KEK, Tsukuba, Ibaraki 305-0801, Japan \\ ${ }^{2}$ The Graduate University of Advanced Studies (Sokendai), \\ Tsukuba, Ibaraki 305-0801, Japan \\ ${ }^{3}$ Rudolf Peierls Centre for Theoretical Physics, The University of Oxford, \\ 1 Keble Road, Oxford OX1 3NP, United Kingdom
}

(Received 27 September 2017; revised manuscript received 25 October 2018; published 12 December 2018)

\begin{abstract}
In this paper, we discuss the Higgs vacuum stability around evaporating black holes. We provide a new approach to investigate the false vacuum decay around the black hole and clearly show how vacuum fluctuations of the Higgs induce a gravitational collapse of the vacuum. Furthermore, we point out that the backreaction of the Hawking radiation cannot be ignored and the gravitational vacuum decay is exponentially suppressed. However, a large number of the evaporating (or evaporated) primordial black holes threaten the existence of the Universe and we obtain a new upper bound on the evaporating primordial black hole abundance from the vacuum stability. Finally, we show that the high-order corrections of the beyond standard model or quantum gravity would not destabilize the Higgs potential, otherwise even a single evaporating black hole triggers a collapse of the electroweak vacuum.
\end{abstract}

DOI: $10.1103 /$ PhysRevD.98.123509

\section{INTRODUCTION}

In the late 1970s, Hawking [1] showed that black holes emit thermal radiation at the Hawking temperature $T_{\mathrm{H}}=$ $1 /\left(8 \pi M_{\mathrm{BH}}\right)$ due to quantum particle creation on strong gravitational field ${ }^{1}$ where $M_{\mathrm{BH}}$ is the black-hole mass. The quantum particle creation around the black hole determines the fate of the evaporating black hole which is still unknown and closely related with the information loss puzzle [2], and furthermore, leads to the spontaneous symmetry restoration [3] or the false vacuum decay around the black hole [4-6] which bring cosmological singular possibility. The latter case can also be interpreted as the catalysis induced by vacuum fluctuations around the black hole.

One of the most curious puzzles of the observed Higgs boson at the LHC experiment [7-10] is that the effective Higgs potential develops an instability at the scale

\footnotetext{
*kohri@ post.kek.jp

†matshiro@post.kek.jp

${ }^{1}$ In the present paper we use natural units where $\hbar=c=$ $k_{B}=G=1$.

Published by the American Physical Society under the terms of the Creative Commons Attribution 4.0 International license. Further distribution of this work must maintain attribution to the author(s) and the published article's title, journal citation, and DOI. Funded by SCOAP ${ }^{3}$.
}

$\Lambda_{I} \approx 10^{11} \mathrm{GeV}^{2}$ where we assume no corrections of the beyond standard model (BSM) and the quantum gravity (QG) [19-22]. Thus, the current electroweak vacuum state of the Universe is not stable, and finally collapses through quantum tunneling [23-25] although the timescale of the decay is longer than the age of our Universe [26-29]. However, vacuum fluctuations induced by the strong gravitational field drastically change the stability of the electroweak vacuum. The recent works suggest that the electroweak vacuum is generally unstable on various gravitational or cosmological backgrounds, e.g., during inflation [30-40] corresponding to de-Sitter spacetime, after inflation [41-46] and around evaporating black holes [47-53]. In particular, recent discussion about the electroweak vacuum stability around evaporating black holes has been growing. The black holes emitting the Hawking radiation reduce the mass and finally evaporate. At the final stage of evaporating black holes, the Hawking temperature is extremely high and the influence of the vacuum stability can not be ignored [52-55]. Generally, there are no mechanisms to prevent the formation of such small black holes which finally evaporates during the history of the

\footnotetext{
${ }^{2}$ The instability scale $\Lambda_{I}$ is generally determined by the value of the Higgs boson mass $m_{h}$ and the top quark mass $m_{t}$. The current observed masses of the Higgs boson $m_{h}=125.09 \pm$ 0.21 (stat) \pm 0.11 (syst) $\mathrm{GeV}[7-10]$ and the top quark $m_{t}=$ $172.44 \pm 0.13$ (stat) \pm 0.47 (syst) GeV [11] suggest the instability scale to be $\Lambda_{I} \approx 10^{11} \mathrm{GeV}$ [12] although this instability scale $\Lambda_{I}$ has the gauge dependence (see [13-18] for the detail).
} 
Universe. In particular, the primordial black holes $(\mathrm{PBH})$ formed by large density fluctuations in the early Universe [56-58] are incompatible with the Higgs vacuum stability $[54,59]$. These phenomena could impose serious constraints on the cosmology or the particle physics beyond the standard model.

However, there is some controversy about how evaporating black holes trigger off the Higgs vacuum decay. The analysis [47-49] of the false vacuum decay around black holes by the Coleman-De Luccia (CDL) instanton formalism [60] obscure the dependence of the quantum particle creation around the black hole. Furthermore, the back-reaction of the Hawking radiation with $T_{\mathrm{H}}=$ $1 /\left(8 \pi M_{\mathrm{BH}}\right)$ can not be ignored as the de Sitter spacetime [61], and it is reasonable intuitively to assume the Higgs potential [62-66] at finite temperature instead of the zerotemperature in the environment of the thermal Hawking flux. The thermal corrections can generally stabilize the Higgs potential (see Fig. 1), and the possibility of the Higgs vacuum decay around black holes would be expected to be lower than what was considered. Besides the backreaction issues of the Hawking radiation, the CDL instanton formalism is physically obscure, and therefore, one needs an another derivation of the decay rate without relying on instanton methods. ${ }^{3}$ In de Sitter space it is well known that one can get the probability of tunneling to true vacuum by two-point correlation function $\left\langle\delta \phi^{2}\right\rangle$ [68]. The decay probability using the two-point correlation function which describes the vacuum fluctuations in quantum field theory (QFT) precisely match the Euclidean correct results [69-71].

In accordance to this sprit we investigate how vacuum fluctuations around black holes trigger off the Higgs vacuum decay by using the two-point correlation function $\left\langle\delta \phi^{2}\right\rangle$. We discuss the renormalized expression of the correlation function $\left\langle\delta \phi^{2}\right\rangle_{\text {ren }}$ [72] for three possible vacua which are Boulware, Unruh, and Hartle-Hawking vacuum in Schwarzschild spacetime, and confirm that the Unruh vacuum is an appropriate vacuum which describes evaporating black holes. By using the renormalized two-point correlation function $\left\langle\delta \phi^{2}\right\rangle_{\text {ren }}$ in Unruh vacuum we provide a new approach to investigate the false vacuum decay around evaporating black holes and apply the Higgs vacuum stability. Furthermore we clearly show that the backreaction of the Hawking radiation cannot be physically ignored and the vacuum fluctuations stabilize the effective Higgs potential. Finally, based on the consideration, we discuss cosmological constraints on the PBHs due to the vacuum stability of the Higgs. We find that just one evaporating $\mathrm{PBH}$ does not cause a collapse of the electroweak vacuum, but on the other hand a large number of PBHs is real serious. In this paper, we provide a

\footnotetext{
${ }^{3}$ The stochastic approach of the false vacuum decay in the black hole background was investigated by [67].
}

quantitative description of the Higgs vacuum stability around evaporating black holes and get a new bound of the PBHs.

The organization of this paper is as follows. In Sec. II, we introduce renormalized vacuum fluctuations for various vacua in Schwarzschild spacetime. In Sec. III, we argue how vacuum fluctuations around evaporating black holes modify the Higgs potential. In Sec. IV, we discuss stabilities of the electroweak vacuum around the block hole and give a cosmological constraint of the PBHs. Section V is devoted to our conclusions and future outlooks.

\section{THE VACUUM FLUCTUATIONS AROUND SCHWARZSCHILD BLACK HOLE}

Formally, quantum effects of the vacuum fluctuations are described by the vacuum expectation value of the energy momentum tensor $\left\langle T_{\mu \nu}\right\rangle$ or the correlation function $\left\langle\delta \phi^{2}\right\rangle$ in the quantum field theory (QFT) in curved spacetime. The former $\left\langle T_{\mu \nu}\right\rangle$ provides a exact description of the quantum backreaction on the geometry, and it is crucial to determine the stability of the background spacetime and the fate of the evaporating black hole. The two-point correlation function $\left\langle\delta \phi^{2}\right\rangle$ corresponds to the vacuum fluctuation and plays an essential role in the vacuum stability. But the correlation function usually diverges and unphysical divergences must be eliminated by the regularization and the renormalization method.

The renormalization of vacuum fluctuations for massless scalar field in Schwarzschild spacetime has been well known and analytical estimation is possible. However, the massive case requires complicated numerical calculations. For briefness, in this section, we review the renormalized vacuum fluctuations for the massless case in Boulware, Unruh, and Hartle-Hawking vacuum following [72] and discuss which is an appreciate vacuum state to describe the evaporating black hole.

The metric in the Schwarzschild coordinates (where we ignore the quantum backreaction of the scalar field on the geometry) can be written by

$$
\begin{aligned}
d s^{2}= & -\left(1-\frac{2 M_{\mathrm{BH}}}{r}\right) d t^{2}+\frac{d r^{2}}{1-2 M_{\mathrm{BH}} / r} \\
& +r^{2}\left(d \theta^{2}+\sin ^{2} \theta d \varphi^{2}\right),
\end{aligned}
$$

which covers the exterior region $r>2 M_{\mathrm{BH}}$ of the spacetime where $M_{\mathrm{BH}}$ is the black hoe mass. The above singularity at the horizon $r=2 M_{\mathrm{BH}}$ can be removed by transforming to Kruskal coordinates. By taking the Kruskal coordinates, we obtain the following metric

$d s^{2}=\frac{32 M_{\mathrm{BH}}^{3}}{r} e^{-r / 2 M_{\mathrm{BH}}} d U d V+r^{2}\left(d \theta^{2}+\sin ^{2} \theta d \phi^{2}\right)$,

where these coordinates $U$ and $V$ are formally given by 


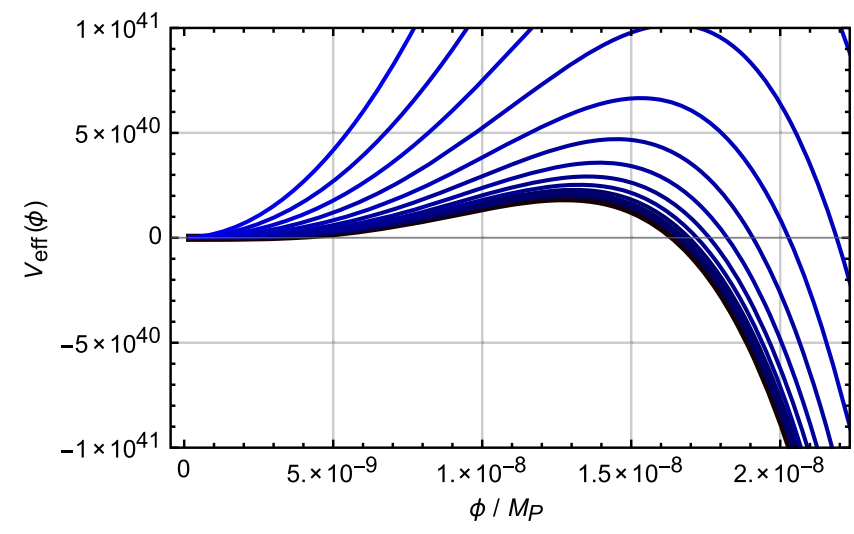

FIG. 1. The schematic diagram of the effective Higgs potential around evaporating black hole. The backreaction of the Hawking radiation around the black hole stabilizes the Higgs potential at $T_{\mathrm{H}} \gtrsim \Lambda_{I}$. The figure sets the present best-fit values of $m_{h}$ and $m_{t}$, and takes different Hawking temperatures $T_{\mathrm{H}}=$ $10^{9.0}-10^{10.2} \mathrm{GeV}$ for the Higgs potential on Eq. (20).

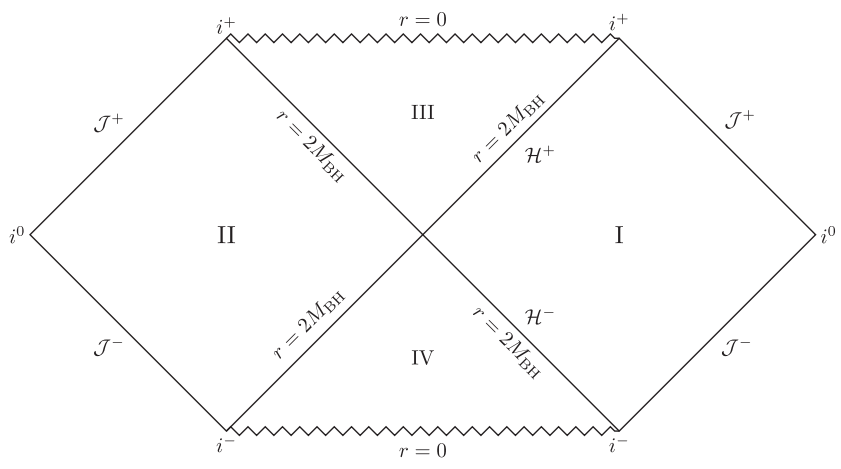

FIG. 2. The Penrose-Carter diagram of the maximally extended Schwarzschild manifold. Regions I or II are asymptotically flat, Region III is the black hole, and Region IV is the white hole. $\mathcal{H}^{+}$ corresponds to the (future) black hole horizon, $\mathcal{H}^{-}$is the (past) black hole horizon, $\mathcal{J}^{+}$corresponds to the (future) null infinity, and $\mathcal{J}^{-}$is the (past) null infinity.

$$
\begin{aligned}
& U=-M_{\mathrm{BH}} e^{-u / 4 M_{\mathrm{BH}}}, \quad V=M_{\mathrm{BH}} e^{v / 4 M_{\mathrm{BH}},} \\
& u=t-r-2 M_{\mathrm{BH}} \ln \left(\frac{r}{2 M_{\mathrm{BH}}}-1\right), \\
& v=t+r+2 M_{\mathrm{BH}} \ln \left(\frac{r}{2 M_{\mathrm{BH}}}-1\right) .
\end{aligned}
$$

The Schwarzschild coordinates of Eq. (1) cover only a part of the spacetime, whereas the Kruskal coordinates of Eq. (2) cover the extended spacetime and is regular at the black hole horizon. These features of the Schwarzschild geometry are summarized in the Penrose-Carter diagrams as Fig. 2.

In curved spacetime, there is no unique vacuum and we must take an appropriate vacuum state. In the Schwarzschild spacetime, there are three well defined vacua, namely:

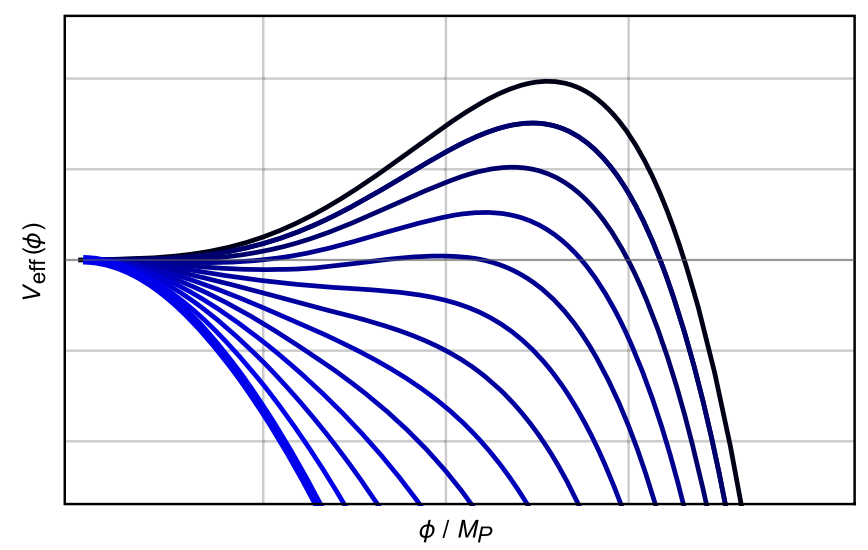

FIG. 3. The schematic diagram of the effective Higgs potential including higher-order corrections of $\lambda_{6}, \lambda_{8}<0$. The backreaction of the Hawking radiation destabilizes the Higgs potential at $T_{\mathrm{H}} \gtrsim \Lambda_{\mathrm{UV}}$ as shown by Eq. (38) and the second-order transition occurs around the black hole.

Boulware vacuum (vacuum state around a static star) [73,74], Unruh vacuum (black hole evaporation) [75], and Hartle-Hawking vacuum (black hole in thermal equilibrium) [76] which correspond to the definitions of the normal ordering on the respective coordinates.

The Klein-Gordon equation for the massless scalar field $\phi(t, x)$ can be given by

$$
\left[-\partial_{\mu} g^{\mu \nu} \sqrt{-g} \partial_{\nu}\right] \phi(t, x)=0
$$

where we drop the curvature term $\xi R \phi^{2}$ because the Ricci scalar becomes $R=0$ in Schwarzschild spacetime ${ }^{4}$ for simplicity, but this approximation may break down when the quantum backreaction on the metric cannot be neglected. In the exterior region of Schwarzschild spacetime, the scalar field $\phi(t, r, \theta, \varphi)$ is decomposed into the from

$$
\begin{aligned}
\phi(t, r, \theta, \varphi)= & \int_{0}^{\infty} d \omega \sum_{l=0}^{\infty} \sum_{m=-l}^{l}\left(a_{\omega l m} u_{\omega l m}^{\mathrm{in}}\right. \\
& \left.+a_{\omega l m}^{\dagger} u_{\omega l m}^{\mathrm{in} *}+b_{\omega l m} u_{\omega l m}^{\mathrm{out}}+b_{\omega l m}^{\dagger} u_{\omega l m}^{\mathrm{out} *}\right),
\end{aligned}
$$

where these mode functions $u_{\omega l m}^{\text {in }}$ and $u_{\omega l m}^{\text {out }}$ define the vacuum state that $a_{\omega l m}|0\rangle=b_{\omega l m}|0\rangle=0$ which corresponds to the boundary conditions. In the Schwarzschild spacetime, these mode functions $u_{\omega l m}^{\text {in }}$ and $u_{\omega l m}^{\text {out }}$ for the massless scalar field are given by

$$
\begin{aligned}
& u_{\omega l m}^{\text {in }}=(4 \pi \omega)^{-1 / 2} R_{l}^{\text {in }}(r ; \omega) Y_{l m}(\theta, \varphi) e^{-i \omega t}, \\
& u_{\omega l m}^{\text {out }}=(4 \pi \omega)^{-1 / 2} R_{l}^{\text {out }}(r ; \omega) Y_{l m}(\theta, \varphi) e^{-i \omega t},
\end{aligned}
$$

\footnotetext{
${ }^{4}$ The Kretschmann scalar $K$ constructed of two Riemann tensors is nonzero, i.e., $K=R_{a b c d} R^{a b c d}=48 M^{2} / r^{6}$.
} 
where these radial functions $R_{l}^{\text {in }}(r ; \omega)$ and $R_{l}^{\text {out }}(r ; \omega)$ have the well-known asymptotic forms,

$$
\begin{aligned}
R_{l}^{\text {in }}(r ; \omega) \simeq \begin{cases}B_{l}(\omega) r^{-1} e^{-i \omega r_{*}} & \left(r \rightarrow 2 M_{\mathrm{BH}}\right) \\
r^{-1} e^{-i \omega r_{*}}+A_{l}^{\mathrm{in}}(\omega) r^{-1} e^{i \omega r_{*}} & (r \rightarrow \infty)\end{cases} \\
R_{l}^{\text {out }}(r ; \omega) \simeq \begin{cases}r^{-1} e^{i \omega r_{*}}+A_{l}^{\text {out }}(\omega) r^{-1} e^{-i \omega r_{*}} & \left(r \rightarrow 2 M_{\mathrm{BH}}\right) \\
B_{l}(\omega) r^{-1} e^{i \omega r_{*}} & (r \rightarrow \infty)\end{cases}
\end{aligned}
$$

$A_{l}^{\text {in }}(\omega), A_{l}^{\text {out }}(\omega)$, and $B_{l}(\omega)$ are the reflection and transmission coefficients [77]. The Boulware vacuum $\left|0_{\mathrm{B}}\right\rangle$ is defined by taking ingoing and outgoing modes to be positive frequency with respect to the Killing vector $\partial_{t}$ of the Schwarzschild metric [73] and constructed by using the scattering theory interpretation.

The two-point correlation function $\left\langle\delta \phi^{2}\right\rangle$ related with the Boulware vacuum $\left|0_{\mathrm{B}}\right\rangle$ can be given by $[72,78]$ :

$$
\begin{aligned}
& \left\langle 0_{\mathrm{B}}\left|\delta \phi^{2}(x)\right| 0_{\mathrm{B}}\right\rangle \\
& =\frac{1}{16 \pi^{2}} \int_{0}^{\infty} \frac{d \omega}{\omega}\left[\sum_{l=0}^{\infty}(2 l+1)\left[\left|R_{l}^{\mathrm{in}}(r ; \omega)\right|^{2}+\left|R_{l}^{\text {out }}(r ; \omega)\right|^{2}\right]\right],
\end{aligned}
$$

where the sum of these radial functions $R_{l}^{\mathrm{in}}(r ; \omega)$ and $R_{l}^{\text {out }}(r ; \omega)$ have the asymptotic forms,

$$
\begin{aligned}
\sum_{l=0}^{\infty}(2 l+1)\left|R_{l}^{\mathrm{in}}(r ; \omega)\right|^{2} & \sim\left\{\begin{array}{l}
\frac{\sum_{l=0}^{\infty}(2 l+1)\left|B_{l}(\omega)\right|^{2}}{4 M_{\mathrm{BH}}^{2}}\left(r \rightarrow 2 M_{\mathrm{BH}}\right), \\
4 \omega^{2}(r \rightarrow \infty)
\end{array}\right. \\
\sum_{l=0}^{\infty}(2 l+1)\left|R_{l}^{\text {out }}(r ; \omega)\right|^{2} & \sim\left\{\begin{array}{l}
\frac{4 \omega^{2}}{1-2 M_{\mathrm{BH}} / r}\left(r \rightarrow 2 M_{\mathrm{BH}}\right) \\
\frac{\sum_{l=0}^{\infty}(2 l+1)\left|B_{l}(\omega)\right|^{2}}{r^{2}}(r \rightarrow \infty)
\end{array} .\right.
\end{aligned}
$$

Thus, the two-point correlation function $\left\langle\delta \phi^{2}\right\rangle$ of Eq. (6) has clear divergences and must be regularized. There are several regularization methods to eliminate the divergences in the quantum field theory (QFT), but the point-splitting regularization is an extremely powerful and standard method to obtain the renormalized expression in curved spacetime. Let us consider temporarily $\delta \phi^{2}(x) \rightarrow \delta \phi(x) \delta \phi\left(x^{\prime}\right)$ to remove the divergences and afterwards take the coincident limit $x^{\prime} \rightarrow x$,

$$
\left\langle\delta \phi^{2}(x)\right\rangle_{\mathrm{ren}}=\lim _{x^{\prime} \rightarrow x}\left[\left\langle\delta \phi(x) \delta \phi\left(x^{\prime}\right)\right\rangle-\left\langle\delta \phi(x) \delta \phi\left(x^{\prime}\right)\right\rangle_{\mathrm{div}}\right],
$$

where $\left\langle\delta \phi(x) \delta \phi\left(x^{\prime}\right)\right\rangle_{\text {div }}$ express the divergence part and is namely the DeWitt-Schwinger counterterm, which can be generally given by [79]

$$
\begin{aligned}
& \left\langle\delta \phi(x) \delta \phi\left(x^{\prime}\right)\right\rangle_{\mathrm{div}} \\
& =\frac{1}{8 \pi^{2} \sigma}+\frac{m^{2}+(\xi-1 / 6) R}{8 \pi^{2}}\left[\gamma+\frac{1}{2} \ln \left(\frac{\mu^{2} \sigma}{2}\right)\right] \\
& \quad-\frac{m^{2}}{16 \pi^{2}}+\frac{1}{96 \pi^{2}} R_{\alpha \beta} \frac{\sigma^{; \alpha} \sigma^{; \beta}}{\sigma},
\end{aligned}
$$

where $\sigma$ is the biscalar associated with the short geodesic, $R$ or $R_{\alpha \beta}$ are respectively the Ricci scalar or tensor and $\gamma$ express the Euler-Mascheroni constant. The renormalization parameter $\mu$ corresponds to the mass $m$ of the scalar field and the massless case lead to the well-known ambiguity [80], but the renormalization procedure can eliminate this ambiguity for $\left\langle T_{\mu \nu}\right\rangle$ by the cosmological experiment or observation. In the Schwarzschild metric for the massless scalar field where $m=0$ and $R=0$, we can simplify the DeWitt-Schwinger counterterm of $\left\langle\delta \phi(x) \delta \phi\left(x^{\prime}\right)\right\rangle_{\text {div }}$ to be,

$$
\left\langle\delta \phi(x) \delta \phi\left(x^{\prime}\right)\right\rangle_{\mathrm{div}}=\frac{1}{8 \pi^{2} \sigma} .
$$

For simplicity we take the time separation $\epsilon$ between $x=(t, r, \theta, \varphi)$ and $x^{\prime}=(t+\epsilon, r, \theta, \varphi)$ and the renormalized expression of $\left\langle\delta \phi^{2}\right\rangle$ in the Boulware vacuum $\left|0_{\mathrm{B}}\right\rangle$ is given by

$$
\begin{aligned}
\left\langle\delta \phi^{2}(x)\right\rangle_{\text {ren }}= & \lim _{\epsilon \rightarrow 0}\left[\frac { 1 } { 1 6 \pi ^ { 2 } } \int _ { 0 } ^ { \infty } \frac { e ^ { - i \omega \epsilon } } { \omega } \left[\sum_{l=0}^{\infty}(2 l+1)\left|R_{l}^{\text {in }}(r ; \omega)\right|^{2}\right.\right. \\
& \left.\left.+\left|R_{l}^{\text {out }}(r ; \omega)\right|^{2}\right] d \omega-\frac{1}{8 \pi^{2} \sigma(\epsilon)}\right] .
\end{aligned}
$$

Taking a second-order geodesic expansion we get the following expression [79]

$$
\sigma(\epsilon)=-\frac{1-2 M_{\mathrm{BH}} / r}{2} \epsilon^{2}-\frac{M_{\mathrm{BH}}^{2}\left(1-2 M_{\mathrm{BH}} / r\right)}{24 r^{4}} \epsilon^{4}+O\left(\epsilon^{5}\right),
$$

where $\epsilon^{-2}$ satisfy the following relation

$$
\epsilon^{-2}=-\int_{0}^{\infty} \omega e^{i \omega \epsilon} d \omega
$$

By using Eqs. (10)-(12), we obtain the renormalized expression of the Boulware vacuum $\left|0_{\mathrm{B}}\right\rangle$ as follows [72],

$$
\begin{aligned}
\left\langle 0_{\mathrm{B}}\left|\delta \phi^{2}(x)\right| 0_{\mathrm{B}}\right\rangle_{\text {ren }}= & \frac{1}{16 \pi^{2}} \int_{0}^{\infty} \frac{d \omega}{\omega}\left[\sum_{l=0}^{\infty}(2 l+1)\left[\left|R_{l}^{\mathrm{in}}(r ; \omega)\right|^{2}+\left|R_{l}^{\mathrm{out}}(r ; \omega)\right|^{2}\right]-\frac{4 \omega^{2}}{1-2 M_{\mathrm{BH}} / r}\right] \\
& -\frac{M_{\mathrm{BH}}^{2}}{48 \pi^{2} r^{4}\left(1-2 M_{\mathrm{BH}} / r\right)} .
\end{aligned}
$$


For the Boulware vacuum $\left|0_{\mathrm{B}}\right\rangle$ we have the asymptotic expression of the renormalized vacuum fluctuations $\left\langle\delta \phi^{2}\right\rangle_{\text {ren }}[72]$

$$
\begin{aligned}
& \left\langle 0_{\mathrm{B}}\left|\delta \phi^{2}(x)\right| 0_{\mathrm{B}}\right\rangle_{\text {ren }} \rightarrow \infty\left(r \rightarrow 2 M_{\mathrm{BH}}\right), \\
& \left\langle 0_{\mathrm{B}}\left|\delta \phi^{2}(x)\right| 0_{\mathrm{B}}\right\rangle_{\text {ren }} \rightarrow 1 / r^{2}(r \rightarrow \infty),
\end{aligned}
$$

where the renormalized expression $\left\langle\delta \phi^{2}\right\rangle_{\text {ren }}$ is singular on the event horizons $r=2 M_{\mathrm{BH}}$ and ill-defined around the black-hole horizon. In the case of the energy momentum tensor $\left\langle T_{\mu \nu}\right\rangle$, the renormalized expression of the energy momentum tensor $\left\langle T_{\mu \nu}\right\rangle_{\text {ren }}$ has been given by Refs. [72,80-85] and shows similar properties to the renormalized expression of $\left\langle\delta \phi^{2}\right\rangle_{\text {ren }} \cdot{ }^{5}$ This state closely reproduces the Minkowski vacuum $\left|0_{\mathrm{M}}\right\rangle$ at infinity because $\left\langle 0_{\mathrm{B}}\left|\delta \phi^{2}\right| 0_{\mathrm{B}}\right\rangle \rightarrow 1 / r^{2}$ in the limit $r \rightarrow \infty$. However, the Boulware vacuum is singular on the event horizon $r=2 M_{\mathrm{BH}}$ and hence unacceptable near the black-hole horizon. Thus, the usual interpretation of the above result is that the Boulware vacuum $\left|0_{\mathrm{B}}\right\rangle$ is considered to be the appropriate vacuum state around a static star and not a black hole.

Next, let us consider the Unruh vacuum $\left|0_{U}\right\rangle$ which is formally defined by taking ingoing modes to be positive frequency with respect to $\partial_{t}$, but outgoing modes to be positive frequency with respect to the Kruskal coordinate $\partial_{U}$ [75]. For the Unruh vacuum we obtain the two-point correlation function $\left\langle\delta \phi^{2}\right\rangle[72,78]$,

$$
\left\langle 0_{\mathrm{U}}\left|\delta \phi^{2}(x)\right| 0_{\mathrm{U}}\right\rangle=\frac{1}{16 \pi^{2}} \int_{0}^{\infty} \frac{d \omega}{\omega}\left[\sum_{l=0}^{\infty}(2 l+1)\left[\left|R_{l}^{\text {in }}(r ; \omega)\right|^{2}+\operatorname{coth}\left(\frac{\pi \omega}{\kappa}\right)\left|R_{l}^{\text {out }}(r ; \omega)\right|^{2}\right]\right]
$$

where we introduce $\kappa=\left(4 M_{\mathrm{BH}}\right)^{-1}$ which is the surface gravity of the black hole and the factor of $\operatorname{coth}\left(\frac{\pi \omega}{\kappa}\right)$ originates from the thermal features of the outgoing modes. The renormalized vacuum fluctuations in the Unruh vacuum $\left|0_{\mathrm{U}}\right\rangle$ are given by

$$
\begin{aligned}
\left\langle 0_{\mathrm{U}}\left|\delta \phi^{2}(x)\right| 0_{\mathrm{U}}\right\rangle_{\mathrm{ren}}= & \frac{1}{16 \pi^{2}} \int_{0}^{\infty} \frac{d \omega}{\omega}\left[\sum_{l=0}^{\infty}(2 l+1)\left[\left|R_{l}^{\mathrm{in}}(r ; \omega)\right|^{2}+\operatorname{coth}\left(\frac{\pi \omega}{\kappa}\right)\left|R_{l}^{\text {out }}(r ; \omega)\right|^{2}\right]\right. \\
& \left.-\frac{4 \omega^{2}}{1-2 M_{\mathrm{BH}} / r}\right]-\frac{M_{\mathrm{BH}}^{2}}{48 \pi^{2} r^{4}\left(1-2 M_{\mathrm{BH}} / r\right)}
\end{aligned}
$$

For the Unruh vacuum $\left|0_{\mathrm{U}}\right\rangle$, we can get an asymptotic expression of the renormalized vacuum fluctuations,

$$
\begin{aligned}
\left\langle 0_{\mathrm{U}}\left|\delta \phi^{2}(x)\right| 0_{\mathrm{U}}\right\rangle_{\mathrm{ren}} & \rightarrow \frac{1}{192 \pi^{2} M_{\mathrm{BH}}^{2}}-\frac{1}{32 \pi^{2} M_{\mathrm{BH}}^{2}} \int_{0}^{\infty} \frac{d \omega \omega \sum_{l=0}^{\infty}(2 l+1)\left|B_{l}(\omega)\right|^{2}}{\omega\left(e^{2 \pi \omega / \kappa}-1\right)} \quad\left(r \rightarrow 2 M_{\mathrm{BH}}\right), \\
\left\langle 0_{\mathrm{U}}\left|\delta \phi^{2}(x)\right| 0_{\mathrm{U}}\right\rangle_{\mathrm{ren}} & \rightarrow 1 / r^{2} \quad(r \rightarrow \infty) .
\end{aligned}
$$

${ }^{5}$ For the Boulware vacuum $\left|0_{\mathrm{B}}\right\rangle$ the renormalized energy momentum tensor $\left\langle T_{\mu \nu}\right\rangle_{\text {ren }}$ is given by

$$
\begin{aligned}
\left\langle 0_{\mathrm{B}}\left|T_{\mu}^{\nu}\right| 0_{\mathrm{B}}\right\rangle_{\text {ren }} \rightarrow & -\frac{1}{30 \cdot 2^{12} \pi^{2} M_{\mathrm{BH}}^{4}\left(1-2 M_{\mathrm{BH}} / r\right)^{2}} \\
& \times\left(\begin{array}{cccc}
-1 & 0 & 0 & 0 \\
0 & 1 / 3 & 0 & 0 \\
0 & 0 & 1 / 3 & 0 \\
0 & 0 & 0 & 1 / 3
\end{array}\right) \quad\left(r \rightarrow 2 M_{\mathrm{BH}}\right), \\
\left\langle 0_{\mathrm{B}}\left|T_{\mu}^{\nu}\right| 0_{\mathrm{B}}\right\rangle_{\text {ren }} \rightarrow 1 / r^{6} \quad(r \rightarrow \infty), &
\end{aligned}
$$

where the renormalized expression of $\left\langle T_{\mu \nu}\right\rangle_{\text {ren }}$ for $\left|0_{\mathrm{B}}\right\rangle$ produces a negative energy density divergence at the black-hole horizon $r=2 M_{\mathrm{BH}}$. This fact originates from the infinite blueshift of the negative energy. which corresponds to the evaporating black hole. The Unruh vacuum $\left|0_{U}\right\rangle$ corresponds to the state where the black hole radiates at the Hawking temperature $T_{\mathrm{H}}=$ $1 /\left(8 \pi M_{\mathrm{BH}}\right)$ in the empty space, and therefore, the vacuum fluctuations $\left\langle\delta \phi^{2}\right\rangle$ approach the thermal fluctuations near the black-hole horizon to be $\left\langle 0_{\mathrm{U}}\left|\delta \phi^{2}\right| 0_{\mathrm{U}}\right\rangle \rightarrow$ $\mathcal{O}\left(T_{\mathrm{H}}^{2}\right)$ in the limit $r \rightarrow 2 M_{\mathrm{BH}}$. Thus, the Unruh vacuum is considered to be an appropriate vacuum which describe the evaporating black hole formed by gravitational collapse.

Finally, we discuss the Hartle-Hawking vacuum $\left|0_{\mathrm{HH}}\right\rangle$ which is formally defined by taking ingoing modes to be positive frequency with respect to $\partial_{V}$, and outgoing modes to be positive frequency with respect to the Kruskal coordinate $\partial_{U}[76]$. In the Hartle-Hawking vacuum $\left|0_{\mathrm{HH}}\right\rangle$, we can get, 


$$
\left\langle 0_{\mathrm{HH}}\left|\delta \phi^{2}(x)\right| 0_{\mathrm{HH}}\right\rangle=\frac{1}{16 \pi^{2}} \int_{0}^{\infty} \frac{d \omega}{\omega}\left[\operatorname{coth}\left(\frac{\pi \omega}{\kappa}\right) \sum_{l=0}^{\infty}(2 l+1)\left[\left|R_{l}^{\text {in }}(r ; \omega)\right|^{2}+\left|R_{l}^{\text {out }}(r ; \omega)\right|^{2}\right]\right] .
$$

For $\left|0_{\mathrm{HH}}\right\rangle$, we obtain the asymptotic expression of the renormalized vacuum fluctuations,

$$
\begin{aligned}
\left\langle 0_{\mathrm{HH}}\left|\delta \phi^{2}(x)\right| 0_{\mathrm{HH}}\right\rangle_{\text {ren }} & \rightarrow \frac{1}{192 \pi^{2} M_{\mathrm{BH}}^{2}} \quad\left(r \rightarrow 2 M_{\mathrm{BH}}\right), \\
\left\langle 0_{\mathrm{HH}}\left|\delta \phi^{2}(x)\right| 0_{\mathrm{HH}}\right\rangle_{\mathrm{ren}} & \rightarrow T_{\mathrm{H}}^{2} / 12 \quad(r \rightarrow \infty),
\end{aligned}
$$

where $\left\langle\delta \phi^{2}\right\rangle_{\text {ren }}$ becomes exactly the thermal fluctuation at infinity, i.e., $\left\langle 0_{\mathrm{HH}}\left|\delta \phi^{2}\right| 0_{\mathrm{HH}}\right\rangle \rightarrow T_{\mathrm{H}}^{2} / 12$ in the limit $r \rightarrow \infty$. Thus, the Hartle-Hawking vacuum corresponds to a black hole in thermal equilibrium at $T_{\mathrm{H}}$. If we consider the vacuum fluctuations around the black hole without no thermal radiation, i.e., the temperature of the universe is lower than the Hawking temperature $T_{\mathrm{H}}$, this vacuum state is not adequate to describe the evaporation of the black hole.

The analytic approximations of $\left\langle\delta \phi^{2}\right\rangle_{\text {ren }}$ and $\left\langle T_{\mu \nu}\right\rangle_{\text {ren }}$ in Schwarzschild spacetime for the various vacua (Boulware, Unruh vacuum, and Hartle-Hawking) and the various fields of spin $0,1 / 2$, and 1 has been studied by Refs. [72,80-95]. The renormalized expression of the various fields are proportional to the inverse of the black-hole mass $M_{\mathrm{BH}}$ near the black-hole horizon and approximately approach the thermal fluctuations with the Hawking temperature $T_{\mathrm{H}}$ besides the Boulware vacuum. The quantum effects of the vacuum fluctuations expressed by $\left\langle\delta \phi^{2}\right\rangle_{\text {ren }}$ and $\left\langle T_{\mu \nu}\right\rangle_{\text {ren }}$ determine the vacuum stability and the evaporation of the black hole. We recall that the Unruh vacuum is an appropriate vacuum state describing the vacuum fluctuations around evaporating black holes. Thus, it is reasonable to assume that the vacuum fluctuations of the various field like the Higgs, $W$ and $Z$ bosons and the top quark approximately approach the Hawking thermal fluctuations around the black hole horizon,

$$
\begin{aligned}
\left\langle\delta \phi^{2}\right\rangle_{\text {ren }} & \approx\left\langle\delta W^{2}\right\rangle_{\text {ren }} \approx\left\langle\delta Z^{2}\right\rangle_{\text {ren }} \approx\left\langle\delta t^{2}\right\rangle_{\text {ren }} \\
& \approx \frac{T_{\mathrm{H}}^{2}}{3}-2 T_{\mathrm{H}}^{2} \int_{0}^{\infty} \frac{d \omega \omega \sum_{l=0}^{\infty}(2 l+1)\left|B_{l}(\omega)\right|^{2}}{\omega\left(e^{2 \pi \omega / \kappa}-1\right)} .
\end{aligned}
$$

\section{THE HIGGS POTENTIAL AROUND EVAPORATING BLOCK HOLES}

In this section we consider the standard model (SM) effective Higgs potential $V_{\text {eff }}(\phi)$ around evaporating block holes where $\phi$ is the Higgs field. The one-loop Higgs potential in 't Hooft-Landau gauge and $\overline{\mathrm{MS}}$ scheme is given by [96-98],

$$
\begin{aligned}
V_{\mathrm{eff}}(\phi)= & \rho_{\Lambda}(\mu)+\frac{m_{\phi}^{2}(\mu)}{2} \phi^{2}+\frac{\lambda_{\phi}(\mu)}{4} \phi^{4} \\
& +\sum_{i=W, Z, t, G, H} \frac{n_{i} M_{i}^{4}(\phi)}{64 \pi^{2}}\left[\log \frac{M_{i}^{2}(\phi)}{\mu^{2}}-C_{i}\right],
\end{aligned}
$$

The coefficients $n_{i}$ and $C_{i}$ are given by

$$
\begin{aligned}
& n_{W}=6, \quad n_{Z}=3, \quad n_{t}=-12, \quad n_{G}=3, \quad n_{H}=1, \\
& C_{W}=C_{Z}=5 / 6, \quad C_{t}=C_{G}=C_{H}=3 / 2,
\end{aligned}
$$

and the mass terms $m_{i}^{2}(\phi)$ of the $W$ and $Z$ bosons, the top quark, the Nambu-Goldstone bosons, and the Higgs boson are given by

$$
\begin{aligned}
& m_{W}^{2}=\frac{1}{4} g^{2} \phi^{2}, \quad m_{Z}^{2}=\frac{1}{4}\left[g^{2}+g^{\prime 2}\right] \phi^{2}, \quad m_{t}^{2}=\frac{1}{2} y_{t}^{2} \phi^{2}, \\
& m_{G}^{2}=m_{\phi}^{2}+\lambda_{\phi} \phi^{2}, \quad m_{H}^{2}=m_{\phi}^{2}+3 \lambda_{\phi} \phi^{2},
\end{aligned}
$$

where $g, g^{\prime}, y_{t}$ are the $S U(2)_{L}, U(1)_{Y}$, top Yukawa couplings and $\lambda_{\phi}$ is the Higgs self-coupling. The potential of Eq. (19) express the Higgs effective potential in flat spacetime, not curved spacetime. Actually, we must include the backreaction from the vacuum fluctuations of the various fields into the Higgs potential. The modified effective Higgs potential including the Higgs, $W$ and $Z$ bosons, and top quark vacuum fluctuations can be given by [35]:

$$
\begin{aligned}
V_{\text {eff }}(\phi)= & \rho_{\Lambda}(\mu)+\frac{m_{\phi}^{2}(\mu)}{2} \phi^{2}+\frac{\lambda_{\phi}(\mu)}{4} \phi^{4} \\
& +\frac{3 \lambda(\mu)}{2}\left\langle\delta \phi^{2}\right\rangle_{\text {ren }} \phi^{2}+\frac{g^{2}(\mu)}{8}\left\langle\delta W^{2}\right\rangle_{\text {ren }} \phi^{2} \\
& +\frac{\left[g^{2}(\mu)+g^{\prime 2}(\mu)\right]}{8}\left\langle\delta Z^{2}\right\rangle_{\text {ren }} \phi^{2}+\frac{y_{t}^{2}(\mu)}{4}\left\langle\delta t^{2}\right\rangle_{\text {ren }} \phi^{2} \\
& +\sum_{i=W, Z, t, G, H} \frac{n_{i} M_{i}^{4}(\phi)}{64 \pi^{2}}\left[\log \frac{M_{i}^{2}(\phi)}{\mu^{2}}-C_{i}\right],
\end{aligned}
$$

The Higgs vacuum fluctuations induce the false vacuum decay but the $W$ and $Z$ bosons and the top quark fluctuations stabilize the Higgs potential via the interaction. Now let us rewrite the effective Higgs potential of Eq. (19) by using the Unruh vacuum $\left|0_{\mathrm{U}}\right\rangle$. The renormalized vacuum fluctuations are proportional to the inverse of the black-hole mass $M_{\mathrm{BH}}$ and the Hawking temperature $T_{\mathrm{H}}$. Thus, it is now evident that the effective Higgs potential around evaporating black holes reproduce the thermal case [62-66] and generally is stabilized. The Higgs potential $V_{\text {eff }}(\phi)$ including the vacuum fluctuations of the various fields around evaporating black holes can be approximately written as, 


$$
V_{\mathrm{eff}}(\phi) \simeq \mathcal{O}\left(T_{\mathrm{H}}^{2}\right) \phi^{2}+\frac{\lambda_{\mathrm{eff}}(\phi)}{4} \phi^{4},
$$

where $\lambda_{\text {eff }}(\phi)$ is the effective Higgs self-coupling defined by the effective potential $V_{\text {eff }}(\phi)$ and the maximal field value $\phi_{\max }$ can be given by [42],

$$
\phi_{\max } \approx \mathcal{O}(10) \sqrt{V_{\text {eff }}^{\prime \prime}(\phi)} \approx \mathcal{O}(1 \sim 10) \cdot T_{\mathrm{H}} \gtrsim \Lambda_{I} .
$$

Thus, it turns out that the Hawking radiation generally stabilizes the Higgs potential but it does not mean that the Higgs vacuum is perfectly stable around evaporating black holes. In the next section we discuss the Higgs vacuum stability around the black hole in more detail.

\section{THE FALSE VACUUM DECAY AROUND EVAPORATING BLACK HOLES}

The false vacuum decay is usually analyzed by the Euclidean or bounce solution where one idealize the system that a bubble of true vacuum is surround by the potential wall and false vacuum sea. The created vacuum bubble expands eating other regions of false vacuum and a first order phase transition is complete. The vacuum decay ratio and critical bubble size can be determined by the Euclidean solution or the instanton which capture the catalysis induced by quantum fluctuations around the vacuum [24,25]. The analysis of the false vacuum decay in flat spacetime is completely understood, but it is not in curved spacetime. Taking into account quantum effects of the gravity, induced vacuum fluctuations and gravitational particle production occurs, and the process of the false vacuum decay is expected to be different from what is considered in flat spacetime. In [60], Coleman and de Luccia (CDL) formulate the approach of the false vacuum decay based on the instanton. The CDL instanton simply describes a bubble nucleation in curved spacetime. The decay rate of the vacuum in curved spacetime is given by

$$
\Gamma_{\text {decay }}(\phi)=A \exp (-B),
$$

where $A$ is a prefactor and $B$ is given by the action of the bounce solution. Following [47-49,99], we review the false vacuum decay around the black hole. For simplicity, we introduce two Schwarzschild geometry with different cosmological constants separated by a thin wall tension,

$$
\begin{aligned}
& d s^{2}=f(r) d \tau_{ \pm}^{2}+\frac{d r^{2}}{f(r)}+r^{2} d \Omega^{2}, \\
& f(r) \equiv 1-\frac{2 G M_{ \pm}}{r}-\frac{\Lambda_{ \pm} r^{2}}{3}
\end{aligned}
$$

where $\tau_{ \pm}$are different time coordinates, $M_{ \pm}$are different black hole masses, and $\Lambda_{ \pm}$are different cosmological constants where we simply assume that Higgs false vacuum has $\Lambda_{+}=0$, and true vacuum has $\Lambda_{-}=-3 / \ell^{2}$. In this geometry, the bounce action with the black hole can be given by [99],

$$
\begin{aligned}
B= & \frac{\mathcal{A}_{h}^{+}}{4 G}-\frac{\mathcal{A}_{h}^{-}}{4 G}+\frac{1}{4 G} \oint d \lambda\left\{\left(2 R-6 G M_{+}\right) \dot{\tau}_{+}\right. \\
& \left.-\left(2 R-6 G M_{-}\right) \dot{\tau}_{-}\right\}
\end{aligned}
$$

where $R\left(\tau_{ \pm}\right)$is the bubble radius and $\mathcal{A}_{h}^{ \pm}$are the black hole horizon areas. The prefactor $A$ can be estimated by this bounce action $B$ and the vacuum decay ratio including the black hole can be roughly rewritten as [47],

$$
\Gamma_{\text {decay }}(\phi) \approx\left(\frac{B}{2 \pi}\right)^{1 / 2}\left(G M_{+}\right)^{-1} e^{-B}
$$

where $B\left(M_{+} / M_{\mathrm{P}}, \phi_{\max }\right)$ depend on the black hole mass $M_{+}$and the maximal field value $\phi_{\max }$ of the Higgs. If the black hole mass approaches the Planck mass with the Hawking temperature $T_{\mathrm{H}} \approx M_{\mathrm{P}} \approx 10^{19} \mathrm{GeV}$ where $M_{\mathrm{P}}$ is the Planck mass, a false vacuum decay around black hole is sufficiently enhanced [47]. However, the barrier of the Higgs potential is sufficiently lifted up by the Hawking radiation from Eq. (21). Taking into account the backreaction effects of the Hawking radiation or the vacuum fluctuations around the black hole, the decay rate is exponentially suppressed. Thus, one Planck mass primordial black hole would not cause serious problems on the vacuum stability of the Higgs.

Generally, the analysis of the Euclidean methods or the instanton is standard to investigate the false vacuum decay. However, they are physically obscure and somewhat suspicious in curved spacetime. Recalling that we have no unique vacua in curved spacetime (the Schwarzschild spacetime has three well-defined vacua, Boulware, Unruh, and Hartle-Hawking vacuum), different vacuum states lead to different vacuum fluctuations and different decay ratios. Thus, one needs to understand what kind of process occurs in the vacuum collapse around the black hole and consider another derivation of the vacuum decay rate without relying on the instanton.

Now, we provide another approach to investigate the false vacuum decay around evaporating black holes by using the renormalized two-point correlation function of Eq. (15) in Unruh vacuum. This approach using the twopoint correlation function $\left\langle\delta \phi^{2}\right\rangle$ is consistent with the instanton method [69-71] and extremely simple even in the investigation of the false vacuum decay with taking account of the gravitational effects.

Let us introduce the vacuum decay rate by using the renormalized two-point correlation function. The probability of the local Higgs fields where the vacuum fluctuations $\left\langle\delta \phi^{2}\right\rangle_{\text {ren }}$ exist can be given by [39], 


$$
P(\phi)=\frac{1}{\sqrt{2 \pi\left\langle\delta \phi^{2}\right\rangle_{\text {ren }}}} \exp \left(-\frac{\phi^{2}}{2\left\langle\delta \phi^{2}\right\rangle_{\text {ren }}}\right) .
$$

By using Eq. (26), we can obtain the probability not to exceed the hill of the Higgs potential as follows [39]:

$$
\begin{aligned}
P\left(\phi<\phi_{\max }\right) & \equiv \int_{-\phi_{\max }}^{\phi_{\max }} P\left(\phi,\left\langle\delta \phi^{2}\right\rangle_{\text {ren }}\right) d \phi \\
& =\operatorname{erf}\left(\frac{\phi_{\max }}{\sqrt{2\left\langle\delta \phi^{2}\right\rangle_{\mathrm{ren}}}}\right),
\end{aligned}
$$

where $\phi_{\max }$ is the maximal field value of the Higgs potential. Considering the probability that the localized Higgs fields go into the negative true vacuum, the vacuum decay ratio is estimated to be

$$
\begin{aligned}
\Gamma_{\text {decay }}(\phi) & \equiv P\left(\phi>\phi_{\max }\right)=1-\operatorname{erf}\left(\frac{\phi_{\max }}{\sqrt{2\left\langle\delta \phi^{2}\right\rangle_{\mathrm{ren}}}}\right), \\
& \simeq \frac{\sqrt{2\left\langle\delta \phi^{2}\right\rangle_{\mathrm{ren}}}}{\pi \phi_{\max }} \exp \left(-\frac{\phi_{\max }^{2}}{2\left\langle\delta \phi^{2}\right\rangle_{\mathrm{ren}}}\right) .
\end{aligned}
$$

Then, the constraint from the vacuum decay for the Higgs field is represented by ${ }^{6}$

$$
\mathcal{N}_{\mathrm{PBH}} \cdot \Gamma_{\text {decay }}(\phi) \lesssim 1,
$$

where $\mathcal{N}_{\mathrm{PBH}}$ is the number of the evaporating (or evaporated) primordial black holes during the cosmological history of the Universe. Substituting Eq. (28) into Eq. (29), we can simplify the constraint of the vacuum stability,

$$
\frac{\left\langle\delta \phi^{2}\right\rangle_{\mathrm{ren}}}{\phi_{\max }^{2}} \lesssim \frac{1}{2}\left(\log \mathcal{N}_{\mathrm{PBH}}\right)^{-1}
$$

in order not to induce a vacuum transition due to large Higgs fluctuations. In the Unruh vacuum $\left|0_{U}\right\rangle$ corresponding to the vacuum state around evaporating black holes, $\left\langle\delta \phi^{2}\right\rangle_{\text {ren }}$ approximately take the value of the Hawking temperature $T_{\mathrm{H}}$ near the horizon. However, at the infinity $\left\langle\delta \phi^{2}\right\rangle_{\text {ren }}$ attenuates rapidly and becomes zero. Thus, we obtain the renormalized vacuum fluctuations $\left\langle\delta \phi^{2}\right\rangle_{\text {ren }}$ around evaporating black holes as follows:

$$
\left\langle\delta \phi^{2}\right\rangle_{\mathrm{ren}} \simeq \mathcal{O}\left(10^{-2}-10^{-1}\right) \cdot T_{\mathrm{H}} \quad\left(r \rightarrow 2 M_{\mathrm{BH}}\right) .
$$

However, the most uncertain thing in the stochastic formalism is how to determine the volume factor of $\mathcal{V}$. We took $\mathcal{V}$ to be the volume of the domains in which the vacuum fluctuation $\left\langle\delta \phi^{2}\right\rangle_{\text {ren }}$ governs. By using Eqs. (21), (30),

\footnotetext{
${ }^{6}$ The detail analysis of the Higgs vacuum decay induced by thermal fluctuations was investigated by [42].
}

and (31), we can estimate a constraint on the number of the evaporating primordial black holes as follows

$$
\begin{aligned}
\mathcal{N}_{\mathrm{PBH}} \cdot \Gamma_{\text {decay }}(\phi) & \simeq \frac{\mathcal{N}_{\mathrm{PBH}} \sqrt{2\left\langle\delta \phi^{2}\right\rangle_{\text {ren }}}}{\pi \phi_{\max }} \exp \left(-\frac{\phi_{\text {max }}^{2}}{2\left\langle\delta \phi^{2}\right\rangle_{\text {ren }}}\right) \\
& \approx \mathcal{N}_{\mathrm{PBH}} \cdot e^{-\mathcal{O}\left(10^{2 \sim 3}\right)} \lesssim 1,
\end{aligned}
$$

where the left-hand side shows the number of the primordial black holes $\mathcal{N}_{\mathrm{PBH}}$ within the presently visible part of the Universe which cause the Higgs vacuum collapse. Thus, we obtain a new bound on the number of the evaporating primordial black holes to be $\mathcal{N}_{\mathrm{PBH}} \lesssim \mathcal{O}\left(10^{43-434}\right)$ which is extremely huge to threaten the Higgs metastability.

Next, let us consider the upper bound on the yield of the PBHs $Y_{\mathrm{PBH}} / \equiv n_{\mathrm{PBH}} / s$ as follows,

$$
Y_{\mathrm{PBH}}=\frac{n_{\mathrm{PBH}}}{s}=\frac{\mathcal{N}_{\mathrm{PBH}}}{s_{0} / H_{0}^{3}} \lesssim \mathcal{O}\left(10^{-43}\right),
$$

where $s_{0}$ denotes the entropy density at present $\left[\approx\left(3 \times 10^{-4} \mathrm{eV}\right)^{3}\right]$, and $H_{0}$ is the current Hubble constant $\left(\approx 10^{-33} \mathrm{eV}\right)$. Note that $Y_{\mathrm{PBH}}$ is constant from the formation time to the evaporation time.

It is convenient to transform this bound into an upper bound on $\beta$, which is defined by taking values at the formation of the $\mathrm{PBH}$ to be

$$
\left.\beta \equiv \frac{\rho_{\mathrm{PBH}}}{\rho_{\text {tot }}}\right|_{\text {formation }},
$$

where $\rho_{\mathrm{PBH}}$ and $\rho_{\text {tot }}$ are the energy density of the PBHs and the total energy density of the Universe including the PBHs at the formation, respectively. It is remarkable that $\beta$ means the number of the PBHs per the horizon volume at the formation $\left(\beta \sim n_{\mathrm{PBH}} / H^{3}\right)$. Then we have a relation [100],

$$
\beta \sim 10^{30} \frac{n_{\mathrm{PBH}}}{s}\left(\frac{m_{\mathrm{PBH}}}{10^{15} \mathrm{~g}}\right)^{3 / 2} .
$$

Combining this relation with (33), we obtain ${ }^{7}$

$$
\beta \lesssim \mathcal{O}\left(10^{-21}\right)\left(\frac{m_{\mathrm{PBH}}}{10^{9} \mathrm{~g}}\right)^{3 / 2} .
$$

This bound can be stronger than the known one for $m_{\mathrm{PBH}} \lesssim$ $10^{9} \mathrm{~g}$ [100]. Furthermore, at the final stage of the black hole evaporation, the black-hole mass $M_{\mathrm{BH}}$ becomes extremely small and the Hawking temperature $T_{\mathrm{H}}$ approaches to the Planck scale $M_{\mathrm{P}} \approx 10^{19} \mathrm{GeV}$. Thus, the UV corrections of the beyond standard model (BSM) and the quantum gravity (QG) can not be ignored and undoubtedly contribute to the false vacuum decay around

\footnotetext{
${ }^{7}$ The Hawking temperature is $T_{\mathrm{H}}=1.058\left(10^{13} \mathrm{~g} / \mathrm{m}_{\mathrm{PBH}}\right) \mathrm{GeV}$.
} 
the black hole. When the Hawking temperature approaches to the Planck scale as $T_{\mathrm{H}} \rightarrow M_{\mathrm{P}}$, the contributions of the $\mathrm{BSM}$ and the QG determine the stability of the vacuum. if these physics destabilize the Higgs potential at the high energy, even a single evaporating black hole cause a vacuum decay of the Universe.

Now, we consider the effective Higgs potential including corrections of the BSM and the QG. For convenience we add two higher dimension operators $\phi^{6}$ and $\phi^{8}$ via the UV physics to the Higgs potential as follows,

$$
V_{\mathrm{eff}}(\phi)=\frac{\lambda_{\mathrm{eff}}(\phi)}{4} \phi^{4}+\frac{\lambda_{6}}{6} \frac{\phi^{6}}{\Lambda_{\mathrm{UV}}^{2}}+\frac{\lambda_{8}}{8} \frac{\phi^{8}}{\Lambda_{\mathrm{UV}}^{4}}+\cdots,
$$

where $\lambda_{6}$ and $\lambda_{8}$ are dimensionless coupling constants. These contributions of $\lambda_{6}$ and $\lambda_{8}$ are usually negligible except for the large field excursion $\phi \approx \Lambda_{\mathrm{UV}}$ although they can affect the false vacuum decay through quantum tunneling [19-22]. However, in the final evaporation of the black hole where $T_{\mathrm{H}} \rightarrow M_{\mathrm{Pl}}$, these quantum corrections of $\lambda_{6}$ and $\lambda_{8}$ can not be neglected and have a strong impact on the vacuum stability. As previously discussed in Sec. IV, the effective potential is modified by vacuum fluctuations around the black hole and can be given as,

$$
\begin{aligned}
V_{\text {eff }}(\phi) \simeq & \mathcal{O}\left(T_{\mathrm{H}}^{2}\right) \phi^{2}+\frac{\lambda_{\text {eff }}(\phi)}{4} \phi^{4} \\
& +\frac{\lambda_{6} \cdot \mathcal{O}\left(T_{\mathrm{H}}^{4}\right)}{6 \Lambda_{\mathrm{UV}}^{2}} \phi^{2}+\frac{\lambda_{8} \cdot \mathcal{O}\left(T_{\mathrm{H}}^{6}\right)}{8 \Lambda_{\mathrm{UV}}^{4}} \phi^{2}+\cdots,
\end{aligned}
$$

which destabilizes at $T_{\mathrm{H}} \approx \Lambda_{\mathrm{UV}}$ when these dimensionless couplings are negative $\lambda_{6}, \lambda_{8}<0$. In this case the derivative of the Higgs potential becomes negative as $\partial V_{\text {eff }}(\phi) / \partial \phi<0$, the local Higgs fields around the black hole classically roll down into the negative Planck-scale true vacuum (see Fig. 3) and the Higgs anti-de Sitter (AdS) bubbles whose sizes are about the black-hole horizon are formed. Not all Higgs AdS bubbles threaten the Universe, which highly depends on their evolutions (see Refs. [39,51] for the detailed discussions). However, the Higgs AdS bubbles generally expand eating other regions of the false vacuum and finally consuming the entire Universe. Thus, even a single evaporating black hole within the presently visible part of the Universe can cause a vacuum decay of the Higgs at $T_{\mathrm{H}} \approx \Lambda_{\mathrm{UV}}$ although this possibility strongly depends on the BSM or the QG and the detail of the evaporation of the black hole.

\section{CONCLUSION}

In this paper, we have investigated the electroweak vacuum stability around evaporating black holes. First, following [72] we have confirmed that the Unruh vacuum $\left|0_{\mathrm{U}}\right\rangle$ is an appreciate vacuum state to describe the evaporating black hole. Then, we have provide a new approach to investigate the false vacuum decay around the black hole using the renormalized vacuum fluctuations $\left\langle\delta \phi^{2}\right\rangle_{\text {ren }}$ in the Unruh vacuum $\left|0_{U}\right\rangle$. Clearly, we have shown how vacuum fluctuations of the Higgs field induce a collapse of the electroweak vacuum.

Furthermore, we have pointed out that backreactions of the Hawking thermal radiation cannot be ignored and the Higgs potential is generally stabilized by the vacuum fluctuations around the black hole which was ignored in previous works of the Higgs vacuum stability. Incorporating the backreaction effects and reanalyzing the stability of the Higgs vacuum around the black hole, the stability conditions approximately reproduce the one for the thermal situation. Thus, one evaporating black hole does not cause serious problems on the standard model Higgs vacuum. However, a large number of the evaporating (or evaporated) primordial black holes still threaten the vacuum and we have obtained a new bound on the evaporating $\mathrm{PBH}$ abundance $\beta \lesssim$ $\mathcal{O}\left(10^{-21}\right)\left(m_{\mathrm{PBH}} / 10^{9} \mathrm{~g}\right)^{3 / 2}$ not to induce a collapse of the Universe.

Finally, we have discussed a possibility at the final stage of the evaporation of the black hole where the black-hole mass $M_{\mathrm{BH}}$ becomes extremely small and the Hawking temperature approaches the Planck scale $M_{\mathrm{P}}$. Thus, the Planck scale physics or the BSM directly may intervene and have a strong impact on the vacuum stability around the black holes. Our discussion will be changed if the Planckscale physics or the BSM destabilize the Higgs potential.

\section{ACKNOWLEDGMENTS}

This work of K. K. is supported in part by MEXT KAKENHI No. JP15H05889, No. JP18H04594, and No. JP16H00877, and JSPS KAKENHI No. 26247042 and No. JP1701131.
[1] S. W. Hawking, Commun. Math. Phys. 43, 199 (1975).

[2] S. W. Hawking, Phys. Rev. D 14, 2460 (1976).

[3] S. W. Hawking, Commun. Math. Phys. 80, 421 (1981).

[4] W. A. Hiscock, Phys. Rev. D 35, 1161 (1987).
[5] V. A. Berezin, V. A. Kuzmin, and I. I. Tkachev, Phys. Lett. B 207, 397 (1988).

[6] P. B. Arnold, Nucl. Phys. B346, 160 (1990).

[7] G. Aad et al. (ATLAS and CMS Collaborations), Phys. Rev. Lett. 114, 191803 (2015). 
[8] G. Aad et al. (ATLAS Collaboration), Phys. Lett. B 726, 88 (2013); 734, 406(E) (2014).

[9] S. Chatrchyan et al. (CMS Collaboration), Phys. Rev. D 89, 092007 (2014).

[10] P. P. Giardino, K. Kannike, I. Masina, M. Raidal, and A. Strumia, J. High Energy Phys. 05 (2014) 046.

[11] V. Khachatryan et al. (CMS Collaboration), Phys. Rev. D 93, 072004 (2016).

[12] D. Buttazzo, G. Degrassi, P. P. Giardino, G. F. Giudice, F. Sala, A. Salvio, and A. Strumia, J. High Energy Phys. 12 (2013) 089.

[13] L. Di Luzio and L. Mihaila, J. High Energy Phys. 06 (2014) 079.

[14] A. Andreassen, W. Frost, and M. D. Schwartz, Phys. Rev. D 91, 016009 (2015).

[15] A. Andreassen, W. Frost, and M. D. Schwartz, Phys. Rev. Lett. 113, 241801 (2014).

[16] Z. Lalak, M. Lewicki, and P. Olszewski, Phys. Rev. D 94, 085028 (2016).

[17] J. R. Espinosa, M. Garny, and T. Konstandin, Phys. Rev. D 94, 055026 (2016).

[18] J. R. Espinosa, M. Garny, T. Konstandin, and A. Riotto, Phys. Rev. D 95, 056004 (2017).

[19] V. Branchina and E. Messina, Phys. Rev. Lett. 111, 241801 (2013).

[20] Z. Lalak, M. Lewicki, and P. Olszewski, J. High Energy Phys. 05 (2014) 119.

[21] V. Branchina, E. Messina, and A. Platania, J. High Energy Phys. 09 (2014) 182.

[22] V. Branchina, E. Messina, and M. Sher, Phys. Rev. D 91, 013003 (2015).

[23] I. Yu. Kobzarev, L. B. Okun, and M. B. Voloshin, Yad. Fiz. 20, 1229 (1974) [Sov. J. Nucl. Phys. 20, 644 (1975)].

[24] S. R. Coleman, Phys. Rev. D 15, 2929 (1977); 16, 1248(E) (1977).

[25] C. G. Callan, Jr. and S. R. Coleman, Phys. Rev. D 16, 1762 (1977).

[26] G. Degrassi, S. Di Vita, J. Elias-Miro, J. R. Espinosa, G. F. Giudice, G. Isidori, and A. Strumia, J. High Energy Phys. 08 (2012) 098.

[27] G. Isidori, G. Ridolfi, and A. Strumia, Nucl. Phys. B609, 387 (2001).

[28] J. Ellis, J. R. Espinosa, G. F. Giudice, A. Hoecker, and A. Riotto, Phys. Lett. B 679, 369 (2009).

[29] J. Elias-Miro, J. R. Espinosa, G. F. Giudice, G. Isidori, A. Riotto, and A. Strumia, Phys. Lett. B 709, 222 (2012).

[30] J. R. Espinosa, G. F. Giudice, and A. Riotto, J. Cosmol. Astropart. Phys. 05 (2008) 002.

[31] M. Herranen, T. Markkanen, S. Nurmi, and A. Rajantie, Phys. Rev. Lett. 113, 211102 (2014).

[32] A. Hook, J. Kearney, B. Shakya, and K. M. Zurek, J. High Energy Phys. 01 (2015) 061.

[33] J. Kearney, H. Yoo, and K. M. Zurek, Phys. Rev. D 91, 123537 (2015).

[34] K. Kohri and H. Matsui, J. Cosmol. Astropart. Phys. 08 (2017) 011.

[35] K. Kohri and H. Matsui, arXiv:1704.06884.

[36] O. Czerwinska, Z. Lalak, M. Lewicki, and P. Olszewski, J. High Energy Phys. 10 (2016) 004.
[37] J.-O. Gong and N. Kitajima, Phys. Rev. D 96, 063521 (2017).

[38] W. E. East, J. Kearney, B. Shakya, H. Yoo, and K. M. Zurek, Phys. Rev. D 95, 023526 (2017).

[39] J. R. Espinosa, G. F. Giudice, E. Morgante, A. Riotto, L. Senatore, A. Strumia, and N. Tetradis, J. High Energy Phys. 09 (2015) 174.

[40] A. Joti, A. Katsis, D. Loupas, A. Salvio, A. Strumia, N. Tetradis, and A. Urbano, J. High Energy Phys. 07 (2017) 058 .

[41] M. Herranen, T. Markkanen, S. Nurmi, and A. Rajantie, Phys. Rev. Lett. 115, 241301 (2015).

[42] K. Kohri and H. Matsui, Phys. Rev. D 94, 103509 (2016).

[43] Y. Ema, K. Mukaida, and K. Nakayama, J. Cosmol. Astropart. Phys. 10 (2016) 043.

[44] K. Enqvist, M. Karciauskas, O. Lebedev, S. Rusak, and M. Zatta, J. Cosmol. Astropart. Phys. 11 (2016) 025.

[45] M. Postma and J. van de Vis, J. Cosmol. Astropart. Phys. 05 (2017) 004.

[46] Y. Ema, M. Karciauskas, O. Lebedev, and M. Zatta, J. Cosmol. Astropart. Phys. 06 (2017) 054.

[47] P. Burda, R. Gregory, and I. Moss, Phys. Rev. Lett. 115, 071303 (2015).

[48] P. Burda, R. Gregory, and I. Moss, J. High Energy Phys. 08 (2015) 114.

[49] P. Burda, R. Gregory, and I. Moss, J. High Energy Phys. 06 (2016) 025.

[50] B. Grinstein and C. W. Murphy, J. High Energy Phys. 12 (2015) 063.

[51] N. Tetradis, J. Cosmol. Astropart. Phys. 09 (2016) 036.

[52] C. Cheung and S. Leichenauer, Phys. Rev. D 89, 104035 (2014).

[53] D. Canko, I. Gialamas, G. Jelic-Cizmek, A. Riotto, and N. Tetradis, Eur. Phys. J. C 78, 328 (2018).

[54] D. Gorbunov, D. Levkov, and A. Panin, J. Cosmol. Astropart. Phys. 10 (2017) 016.

[55] K. Mukaida and M. Yamada, Phys. Rev. D 96, 103514 (2017).

[56] S. Hawking, Mon. Not. R. Astron. Soc. 152, 75 (1971).

[57] B. J. Carr and S. W. Hawking, Mon. Not. R. Astron. Soc. 168, 399 (1974).

[58] B. J. Carr, Astrophys. J. 201, 1 (1975).

[59] P. S. Cole and C. T. Byrnes, J. Cosmol. Astropart. Phys. 02 (2018) 019.

[60] S. R. Coleman and F. De Luccia, Phys. Rev. D 21, 3305 (1980).

[61] S. W. Hawking and I. G. Moss, Phys. Lett. B 110, 35 (1982).

[62] P. B. Arnold and S. Vokos, Phys. Rev. D 44, 3620 (1991).

[63] M. E. Carrington, Phys. Rev. D 45, 2933 (1992).

[64] G. W. Anderson and L. J. Hall, Phys. Rev. D 45, 2685 (1992).

[65] C. Delaunay, C. Grojean, and J. D. Wells, J. High Energy Phys. 04 (2008) 029.

[66] L. Delle Rose, C. Marzo, and A. Urbano, J. High Energy Phys. 05 (2016) 050.

[67] S. Iso and S. Okazawa, Nucl. Phys. B851, 380 (2011).

[68] A. D. Linde, D. A. Linde, and A. Mezhlumian, Phys. Rev. D 49, 1783 (1994). 
[69] A. D. Linde, Nucl. Phys. B372, 421 (1992).

[70] A. D. Linde, Rep. Prog. Phys. 42, 389 (1979).

[71] M. Dine, R. G. Leigh, P. Y. Huet, A. D. Linde, and D. A. Linde, Phys. Rev. D 46, 550 (1992).

[72] P. Candelas, Phys. Rev. D 21, 2185 (1980).

[73] D. G. Boulware, Phys. Rev. D 11, 1404 (1975).

[74] D. G. Boulware, Phys. Rev. D 12, 350 (1975).

[75] W. G. Unruh, Phys. Rev. D 14, 870 (1976).

[76] J. B. Hartle and S. W. Hawking, Phys. Rev. D 13, 2188 (1976).

[77] B. S. DeWitt, Phys. Rep. 19, 295 (1975).

[78] S. M. Christensen and S. A. Fulling, Phys. Rev. D 15, 2088 (1977).

[79] S. M. Christensen, Phys. Rev. D 14, 2490 (1976).

[80] P. R. Anderson, W. A. Hiscock, and D. A. Samuel, Phys. Rev. D 51, 4337 (1995).

[81] D. N. Page, Phys. Rev. D 25, 1499 (1982).

[82] M. R. Brown, A. C. Ottewill, and D. N. Page, Phys. Rev. D 33, 2840 (1986).

[83] V. P. Frolov and A. I. Zelnikov, Phys. Rev. D 35, 3031 (1987).

[84] C. Vaz, Phys. Rev. D 39, 1776 (1989).
[85] F. A. Barrios and C. Vaz, Phys. Rev. D 40, 1340 (1989).

[86] K. W. Howard and P. Candelas, Phys. Rev. Lett. 53, 403 (1984).

[87] K. W. Howard, Phys. Rev. D 30, 2532 (1984).

[88] R. M. Nugaev, Phys. Rev. D 43, 1195 (1991).

[89] J. Matyjasek, Phys. Rev. D 53, 794 (1996).

[90] J. Matyjasek, Acta Phys. Pol. B 27, 2005 (1996).

[91] J. Matyjasek, Classical Quantum Gravity 14, L15 (1997). [92] J. Matyjasek, Phys. Rev. D 55, 809 (1997).

[93] M. Visser, Phys. Rev. D 56, 936 (1997).

[94] J. Matyjasek, Phys. Rev. D 57, 7615 (1998).

[95] J. Matyjasek, Phys. Rev. D 59, 044002 (1999).

[96] C. Ford, D. R. T. Jones, P. W. Stephenson, and M. B. Einhorn, Nucl. Phys. B395, 17 (1993).

[97] J. A. Casas, J. R. Espinosa, and M. Quiros, Phys. Lett. B 342, 171 (1995).

[98] J. R. Espinosa and M. Quiros, Phys. Lett. B 353, 257 (1995).

[99] R. Gregory, I. G. Moss, and B. Withers, J. High Energy Phys. 03 (2014) 081.

[100] B. J. Carr, K. Kohri, Y. Sendouda, and J. Yokoyama, Phys. Rev. D 81, 104019 (2010). 